\title{
Transient printing characteristics with wavy build profiles for laser additive manufacturing of small size structures
}

\author{
Fu-qin Liu', *Hui-liang Wei', Lei Wei' ${ }^{2}$, Ting-ting Liu', Wen-he Liao' \\ 1. School of Mechanical Engineering, Nanjing University of Science and Technology, Nanjing 210094, China \\ 2. State Key Laboratory of Solidification Processing, Northwestern Polytechnical University, Xi'an 710072, China
}



\section{*Hui-liang Wei}

Ph.D., Professor. His research interests mainly focus on additive manufacturing of metallic materials. Prof. Wei has made significant progresses in the exploration of process-structureproperties correlations of powder bed fusion and directed energy deposition of diverse materials. He has developed advanced mechanistic and machine learning models to reveal the critical features of various geometrical and metallurgical factors for better printing. $\mathrm{He}$ has hosted and participated in over 10 research projects. He is the Associate Editor of Additive Manufacturing, and also serves on the Editorial Boards of Additive Manufacturing Letters, and Science and Technology of Welding and Joining journals. To date, he has published over 50 peer-reviewed papers.

E-mail: hlwei@njust.edu.cn
Abstract: Laser directed energy deposition (DED) is a multi-physics process that accompanies mass flow, energy transfer, and complex phase transitions. The printing characteristics of small size parts are significantly affected by the progressive variations of the temperature fields and the fluid flow within the molten pool. In this work, the deposition characteristics during multi-layer and multi-track laser DED were explored through a well-tested phenomenological model and corresponding experimental results. The variations of the build profiles and the decoupled track and layer profiles were systematically examined. Moreover, the printing characteristics of the builds with different scanning lengths were compared. Results showed that the multi-layer and multi-track transient deposition processes generated a significantly wavy surface profile. Compared with the long scanning length part, the beginning region of the short build produced an obvious bulge followed by sharply decreased height along the scanning direction. The transverse section of the short build varied significantly at different positions. Two adjacent columns of tracks were extracted from the overall build, demonstrating that the tracks tilted outwards and the angle increases along the scanning direction. The $3 \mathrm{D}$ numerical model was validated with corresponding experiments for builds with various layers. The scientific findings from this work can provide useful insights for the understanding of the additive mechanisms during laser DED for the precise shape control of small size parts.

Key words: additive manufacturing; multi-layer and multi-track; numerical model; transient deposition

CLC numbers: TG146.23; Document code: A; Article ID: 1672-6421 (2021)04-409-07

\section{Introduction}

Laser directed energy deposition (DED) is an additive manufacturing (AM) approach ${ }^{[1-3]}$, which involves a moving laser beam and multiple powder particles to fabricate the desired parts. The build size and forming quality are key factors to be considered during laser DED. These factors are mainly affected by different process parameters, such as the laser power, scanning speed, mass feed rate and scanning strategies ${ }^{[4-6]}$. Since laser DED is a complex process involving transient process variations ${ }^{[7]}$, the study of the progressive printing processes for various structures including the small size parts would be of great significance.

Mechanistic models can help to reveal the spatiotemporal variations of the temperature and fluid flow fields, the freeform build profiles and further the decoupled track and layer profiles. Several comprehensive simulation models were applied to explore the laser DED process ${ }^{[8-11]}$. Knapp et al. ${ }^{[4]}$ utilized a transient, three-dimensional simulation model to explore the temperature and velocity fields, cooling rate and the deposition profile. Wolff et al. ${ }^{[12]}$ considered the combined parameters affecting the shape and size of the molten pool, and presented the thermal history and microhardness in the DED 
process. Except for single track DED, Michopoulos et al. ${ }^{[13]}$ established a multi-physics model to explore the influence of different hatch spacings on the height of molten pool in a single layer multi-track DED. Bai et al. ${ }^{[14]}$ developed a threedimensional model to investigate deposition profile and heat transfer behaviors in the single track multi-layer deposition of plasma arc welding. Huang et al. ${ }^{[15]}$ developed a physicsbased model to predict the size of the molten pool, dilution, process heating/cooling rates and clad 3D profiles from singletrack to multi-track and multi-layer deposition. Besides, the scanning strategy has an important influence on the deposition profile during the wire feeding process ${ }^{[16]}$. The abnormality at the start and end of the weld bead in additive manufacturing with GMAW was explored by $\mathrm{Hu}$ et al ${ }^{[17]}$. Although many microscopic and macroscopic mechanisms of laser DED have been revealed through numerical simulation, the influence of build size on forming quality is still inadequately studied.

Some works have been done in laser DED focusing on the long scanning length deposition. Wei et al. ${ }^{[18]}$ explored the single track, single track multi-layer, and multi-layer multitrack transient laser DED process. They revealed how the process parameters affected the deposition size and profile. It was also found that improper hatch spacing and powder feed rate may cause the formation of the inter-track void ${ }^{[19]}$. The unidirectional scanning strategy may print uneven parts, and the remelting mode between different input parameters was quite different ${ }^{[20]}$. Shrestha et al. ${ }^{[21]}$ explored the transient and steady-state regions from single-track deposition in laser powder bed fusion. It was revealed that the scanning length can greatly affect the profile and size of the deposition. The impact of scanning length on the profile and build size in laser DED has not been sufficiently explored so far. Additionally, the problem of how to avoid the profile defects caused by short-track deposition also needs to be solved.

In this work, the effect of the scanning length on the profile and build size in multi-layer and multi-track laser DED were systematically explored. The deposition track was used to reveal the remelting characteristics of the part. Further, the influence of the deposition length on the final forming quality was revealed by comparing the short and long scanning length builds. The calculated result was validated by experiments. The contents of this work can help to better understand the forming mechanism of small size parts in the laser DED process.

\section{Methodologies}

Laser DED is a multi-physics process that accompanies mass flow, energy transfer, and complex phase transition. In order to make the model more tractable, several assumptions were made ${ }^{[19]}$. The fluid flow in the molten pool was considered laminar, incompressible, and Newtonian. The density of liquid metal was assumed to be a constant. The laser heat flux was assumed to be a Gaussian surface heat source combined with a Gaussian body heat source, and the powder flow was assumed to be a Gaussian distribution. Besides, only the metal powder that interfaces with the molten pool can contribute to metal powder depositing.

An open-source software OpenFOAM ${ }^{[22]}$ was applied. The volume of fluid (VOF) method was employed to capture the gas and metal interface. The details of the approaches can be found in our previous works ${ }^{[18-20]}$, and only the salient features are described here. The model solves the conservation equations of mass, momentum, and energy with the phase equation for VOF. Driving forces for the liquid metal flow in the molten pool including the surface tension, Marangoni stress, and recoil pressure are considered. The radiation, convection and vaporization of heat losses occur at the boundary of the computational domain:

$$
Q_{l}=\left(q_{\text {radLoss }}+q_{\text {convLoss }}+q_{\text {vapLoss }}\right)|\nabla \gamma|
$$

where $Q_{l}$ is the heat loss. $q_{\text {radLoss }}, q_{\text {convLoss }}, q_{\text {vapLoss }}$ are the heat losses caused by radiation, convection, and evaporation, respectively.

$$
\begin{gathered}
q_{\text {radLoss }}=-\sigma_{\mathrm{b}} \varepsilon\left(T^{4}-T_{\text {ref }}^{4}\right) \\
q_{\text {convLoss }}=-h_{\mathrm{c}}\left(T-T_{\text {ref }}\right) \\
q_{\text {vapLoss }}=-\frac{0.82 h_{\mathrm{v}} M}{\sqrt{2 \pi M R_{\mathrm{g}} T}} P_{0} \exp \left[\frac{h_{\mathrm{v}} M\left(T-T_{\mathrm{b}}\right)}{R_{\mathrm{g}} T T_{\mathrm{b}}}\right]
\end{gathered}
$$

where $\sigma_{\mathrm{b}}$ is the Stefan-Boltzmann constant, $\varepsilon$ is the emissivity, $h_{\mathrm{c}}$ is the convective heat transfer coefficient, $T_{\text {ref }}$ is the reference temperature, $h_{\mathrm{v}}$ is the latent heat of vaporization, $T_{\mathrm{b}}$ is the evaporation temperature, $M$ is the molar mass, and $R_{\mathrm{g}}$ is the universal gas constant.

The twenty-layer and six-track, as well as the tenlayer and one-track cases were simulated. The track-wise bidirectional scanning and layer-wise unidirectional scanning strategies were used, and the hatch spacing was $4.2 \mathrm{~mm}$. The unidirectional scanning strategy was used for the ten-layer and one-track case. The process parameters for the laser DED cases are presented in Table 1.

A powder feeding system developed by the Northwestern Polytechnical University was employed, which equiped with the YLS-3000-CL ytterbium laser system, and the DPSF-2 mass feed system. The laser diameter is $3 \mathrm{~mm}$, and the powder feed system includes a side-injection nozzle tip and a 3 -axis working table. The substrate is a pure Ti plate which is $140 \mathrm{~mm}$ long, $50 \mathrm{~mm}$ wide and $6 \mathrm{~mm}$ high. The deposited material is Ti$6 \mathrm{Al}-4 \mathrm{~V}$ (TC4) powder with a size range of 53-150 $\mu \mathrm{m}$. The thermophysical properties of Ti-6Al-4V used in the calculation are presented in Table 2, where $T$ is temperature. The viscosity of the liquid alloy is assumed to be constant in the model ${ }^{[22]}$. Stronger fluid flow and thus more severe geometrical nonuniformity would be generated if lower temperature-dependent viscosity values are used in the model. More details about the experiment system are available in Refs. [20, 23]. 
Table 1: Process parameters of laser DED cases

\begin{tabular}{cccccc} 
Case No. & $\begin{array}{c}\text { Laser power } \\
(\mathbf{W})\end{array}$ & $\begin{array}{c}\text { Scanning speed } \\
\left(\mathbf{m m} \cdot \mathbf{s}^{-1}\right)\end{array}$ & $\begin{array}{c}\text { Powder feed rate } \\
\left(\mathbf{g} \cdot \mathbf{m i n}^{-1}\right)\end{array}$ & $\begin{array}{c}\text { Deposition length } \\
(\mathbf{m m})\end{array}$ & $\begin{array}{c}\text { Laser } \\
\text { scanning strategy }\end{array}$ \\
\hline 1 & 1,800 & 5 & 20 & 25 & Six-track, twenty-layer \\
2 & 1,500 & 8 & 20 & 42 & One-track, ten-layer \\
3 & 1,500 & 8 & 20 & 25 & One-track, ten-layer
\end{tabular}

Table 2: Thermophysical properties of Ti-6Al-4V used in the model ${ }^{[22]}$

\begin{tabular}{|c|c|}
\hline Thermophysical properties & Value \\
\hline Density $\left(\mathrm{kg} \cdot \mathrm{m}^{-3}\right)$ & 4,000 \\
\hline Solidus temperature $(\mathrm{K})$ & 1,878 \\
\hline Liquidus temperature $(\mathrm{K})$ & 1,928 \\
\hline Latent heat of fusion $\left(\mathrm{m}^{2} \cdot \mathrm{s}^{-2}\right)$ & $2.85 \times 10^{5}$ \\
\hline Thermal conductivity of solid $\left(\mathrm{W} \cdot \mathrm{m}^{-1} \cdot \mathrm{K}^{-1}\right)$ & $1.57+2.9 \times 10^{-2} T-7 \times 10^{-6} T^{2}$ \\
\hline Thermal conductivity of liquid $\left(\mathrm{W} \cdot \mathrm{m}^{-1} \cdot \mathrm{K}^{-1}\right)$ & 33 \\
\hline Specific heat of solid $\left(\mathrm{J} \cdot \mathrm{kg}^{-1} \cdot \mathrm{K}^{-1}\right)$ & $512.4+0.15 T-1 \times 10^{-6} T^{2}$ \\
\hline Specific heat of liquid $\left(\mathrm{J} \cdot \mathrm{kg}^{-1} \cdot \mathrm{K}^{-1}\right)$ & 825 \\
\hline Viscosity $\left(\mathrm{kg} \cdot \mathrm{m}^{-1} \cdot \mathrm{s}^{-1}\right)$ & $4 \times 10^{-3}$ \\
\hline $\mathrm{d} \sigma / \mathrm{d} T\left(\mathrm{~N} \cdot \mathrm{m}^{-1} \cdot \mathrm{K}^{-1}\right)$ & $-0.26 \times 10^{-3}$ \\
\hline
\end{tabular}

\section{Results and discussion}

\subsection{Deposition process of DED}

The spatiotemporal variations of temperature field and build profiles during multi-layer and multi-track laser DED are shown in Fig. 1. The black lines show the profile of the molten pool. The powder and substrate experienced a laser heating, melting, solidification and cooling cycle during the laser DED process. The bidirectional scanning strategy was used between different tracks on a layer, and the same scanning strategy was also applied between different layers. It can be found from Fig. 1 that the shape and size of the molten pool change greatly with the increase of layers. Comparing Fig. 1(a) and Fig. 1(d), it can be observed that the size of the molten pool increases with the increasing number of layers. Additionally, the shape changed from puddle-like to saddle-like profiles at higher layers ${ }^{[20]}$. Such a different molten pool shape originates from the continuous deposition characteristics of laser DED and the free flow of the fluid in the molten pool. The phenomenon implies that attention should be paid to the relationship between the transient deposition and the transient shape of the pool.

The flow of fluid in the molten pool is the main reason for the formation of various shapes of parts. The fluid in the molten pool mainly flows backward and sideways ${ }^{[24]}$. Due to the effect of Marangoni convection and surface tension on the fluid in the molten pool, the height difference between the head and tail of the deposition track gradually increases with the increase of layers ${ }^{[19,25]}$. The changes of the whole layer profile will be discussed in the following sections.

\subsection{Characteristics of single track during multi-layer multi-track laser DED}

Figure 2 shows the comparison of transient deposition with a scanning length of $25 \mathrm{~mm}$ and $42 \mathrm{~mm}$ cases. Blue represents the result of the short scanning length $(25 \mathrm{~mm})$ transient deposition, while the red represents the result of the long scanning length $(42 \mathrm{~mm})$ deposition. It can be observed that the bulge of the head is particularly obvious during short scanning length transient deposition, and the height of the deposition track drops sharply along the scanning direction. A similar phenomenon was found in the LPBF (laser powder bed fusion) cases ${ }^{[21]}$. The long scanning length deposition tracks can be divided into three regions: the bulging region at the beginning, the stabilizing region in the middle, and the descending region at the end ${ }^{[20]}$. In addition, it can be observed that the beginning zone of short scanning length transient deposition is higher than the beginning zone of long scanning length transient deposition case, while the final zone is lower than that of the long scanning length deposition. This phenomenon is mainly caused by the complex fluid flow in the molten pool. Meanwhile, with the increase in the number of layers, the height difference between short-track deposits and 

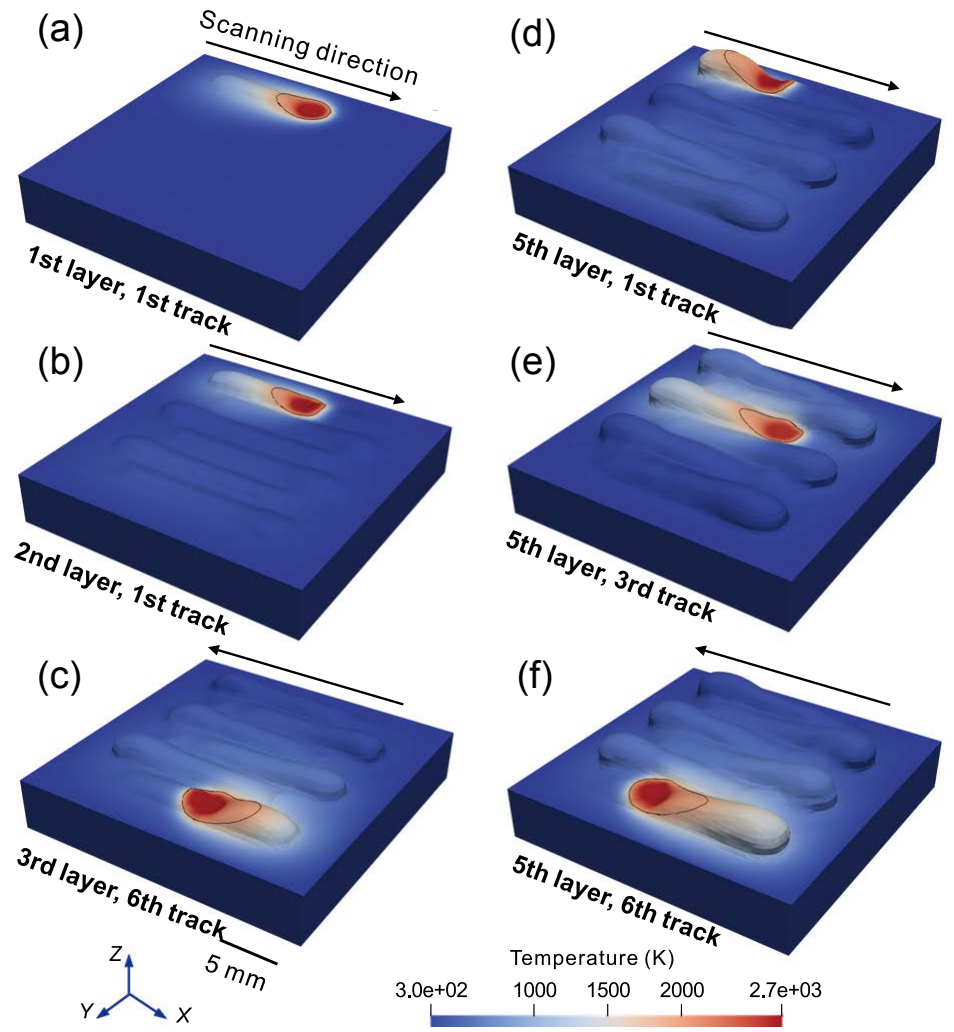

Fig. 1: Variations of temperature field and build profiles during multi-layer and multi-track laser DED

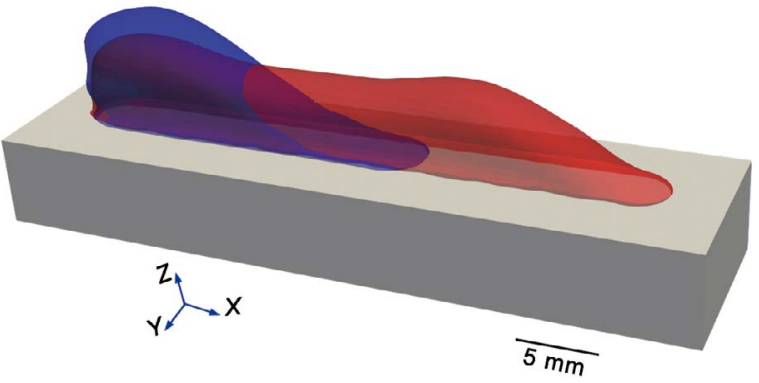

Fig. 2: Comparison of short scanning length $(25 \mathrm{~mm})$ and long scanning length $(42 \mathrm{~mm})$ transient deposition for tenlayer DED with unidirectional laser scanning strategy: laser power $1,500 \mathrm{~W}$, scanning speed $8 \mathrm{~mm} \cdot \mathrm{s}^{-1}$, powder feeding rate $20 \mathrm{~g} \cdot \mathrm{min}^{-1}$

long-track deposits at the beginning stage of the deposition track becomes greater and greater, as shown in Fig. 3. Besides, the slope of the short-track deposition along the scanning direction also increases with the increased number of layers.

Figure 4 shows two adjacent columns extracted from the overall twenty-layer two-track build, which demonstrates the variations of transverse section along the scanning direction. The transverse sections vary greatly from different positions. It can be observed that at the beginning region of the deposition, the transverse section of the deposition track is narrow at the bottom and wide at the top. The middle position is slightly inclined to the lateral side due to the influence of the adjacent deposition track. At the end of the deposition track, there is an obvious incline, and the inner part of the deposition track

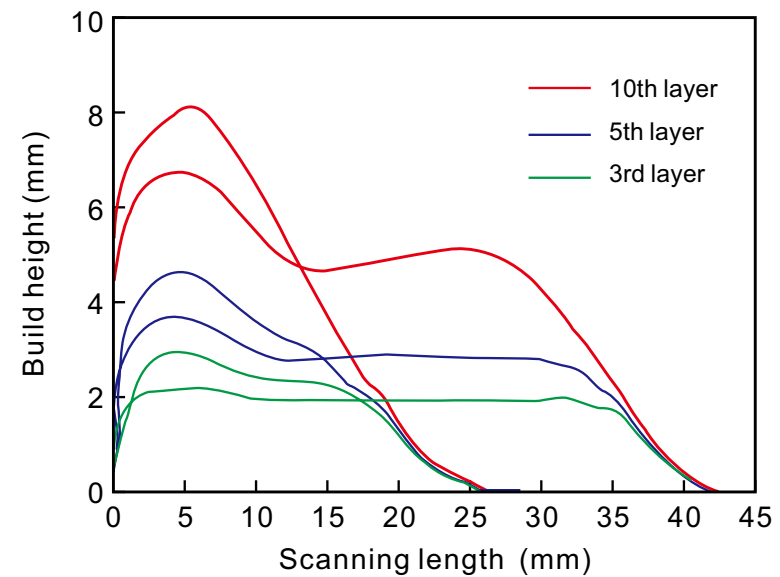

Fig. 3: Variations of deposit height along scanning direction for builds with different scanning lengths
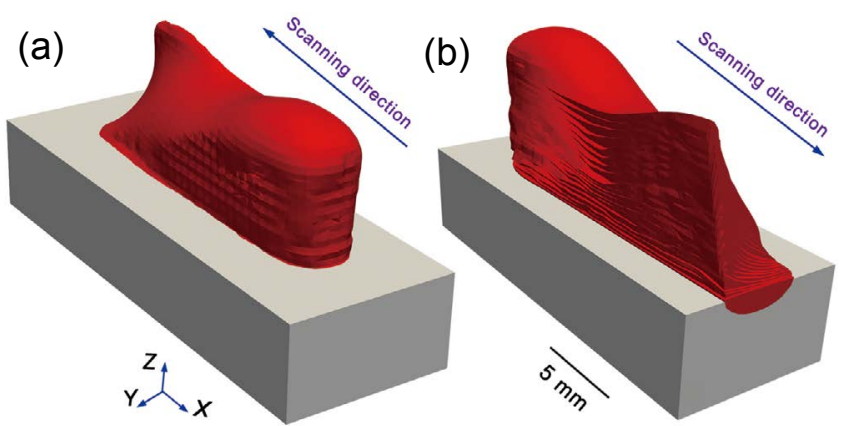

Fig. 4: Printing profiles of decoupled columns of vertical tracks with twenty layers 
is much higher than the outer part. The tiny rough surfaces on the left-hand side of the sample in Fig. 4(a) originated from the dynamic and non-uniform mesh used in the model.

\subsection{Characteristics of transverse section during multi-layer multi-track laser DED}

Figure 5 shows the deposition profile of a single track of different layers, as well as transverse sections at different positions along the build length of different layers. The deposition tracks of the 5th, 10th, 15th, and 20th layers are shown in Fig. 5(a) from the bottom to the top. The first layer is deposited on a flat substrate to form an overall uniform deposition track, and the top surface of the previous layer is used as the substrate for subsequent depositions. The shape of the deposition track undergoes tremendous changes under the combined action of the Marangoni force and the top profile of the previous layer. From the comparison of different layers, it can be seen that as the number of layers increases, the deflection angle of the tail of the deposition track becomes larger and larger. As shown in Fig. 6, the transverse of different positions has different changing trends with the increase of the number of layers. At the position of $x=5 \mathrm{~mm}$, the deflection angle of the transverse section only shows a small change. At the position of $x=12 \mathrm{~mm}$, the angle of transverse section deflection gradually increases, and at the position of $x=20$ $\mathrm{mm}$, there is a sharp increase with the increase of the number of layers. The improper hatch spacing is a paramount factor leading to this phenomenon ${ }^{[18]}$. The proper hatch spacing can produce a dense and uniform part.

The comprehensive simulation model is validated by the corresponding experiment. The comparison of deposited profiles between computed and experimental results is shown in Fig. 7. Both the shape and size of the simulated deposited profile agree well with the experimental result. Figure 7(e) presents the $42 \mathrm{~mm}$ scanning length deposition case. Comparing Fig. 7(e) and Fig. 7(b), it can be found that the long scanning case has a more uniform shape than the short scanning case. The height gap between the head and tail of the short-track deposition is more obvious than that of the long-track deposition, which is similar to the results of the previous single-track multi-layer deposition ${ }^{[20]}$.

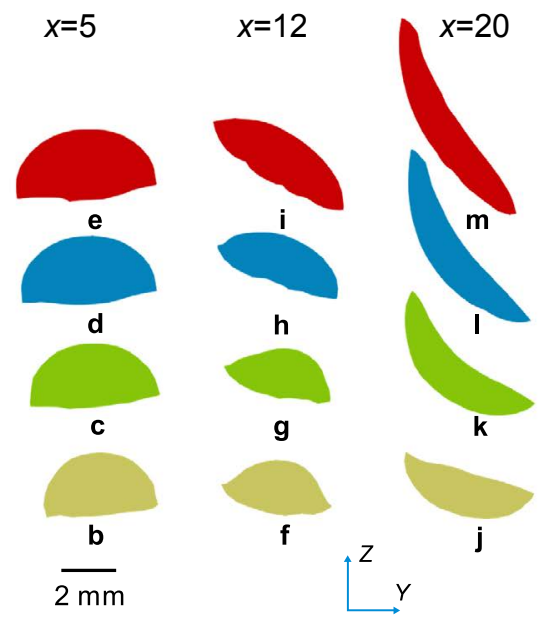

Fig. 5: Deposition profile (a) and transverse sections of different layers: (b)-(e) $x=5 \mathrm{~mm}$, (f)-(i) $x=12 \mathrm{~mm},(\mathrm{j})-(\mathrm{m}) x=20 \mathrm{~mm}$, (b), (f) and (j) fifth layer, (c), (g) and (k) tenth layer, (d), (h) and (l) fifteenth layer, (e), (i) and (m) twentieth layer

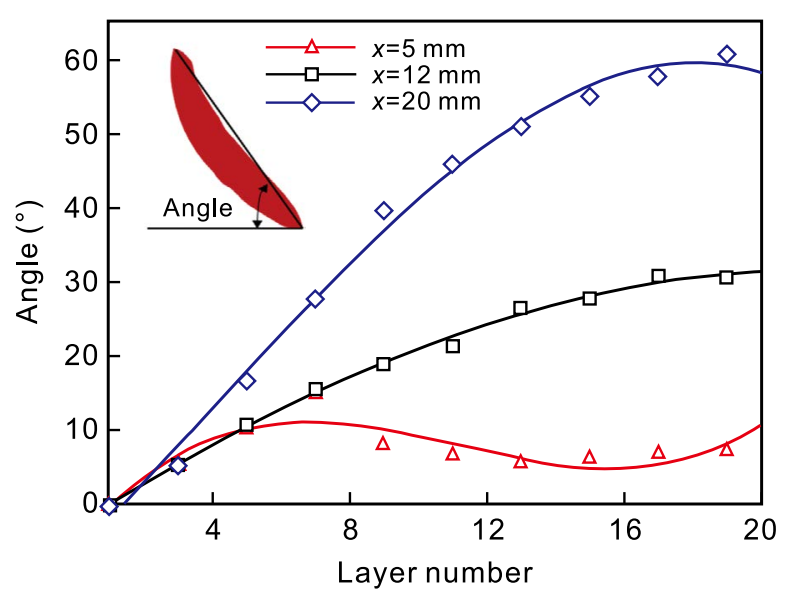

Fig. 6: Changing trend of deflection angle of transverse section along scanning direction of different layers
Figure 8 shows the comparison of deposit profiles between different layers. The long scanning length deposition case has been explored in a previous work ${ }^{[18]}$. Comparing the results of short scanning length deposition with long scanning length deposition cases, it can be observed that the short deposition case at the 10th layer has a wavy shape similar to that of the long deposition case at the 20th layer. As the number of layers increases, the wavy shape becomes more and more obvious. The results prove that the scanning length is also an important factor to affect the forming quality in the laser DED process.

\section{Conclusions}

In this work, the deposition characteristics during multi-layer and multi-track laser DED were explored through a well-tested phenomenological model and corresponding experimental results. 


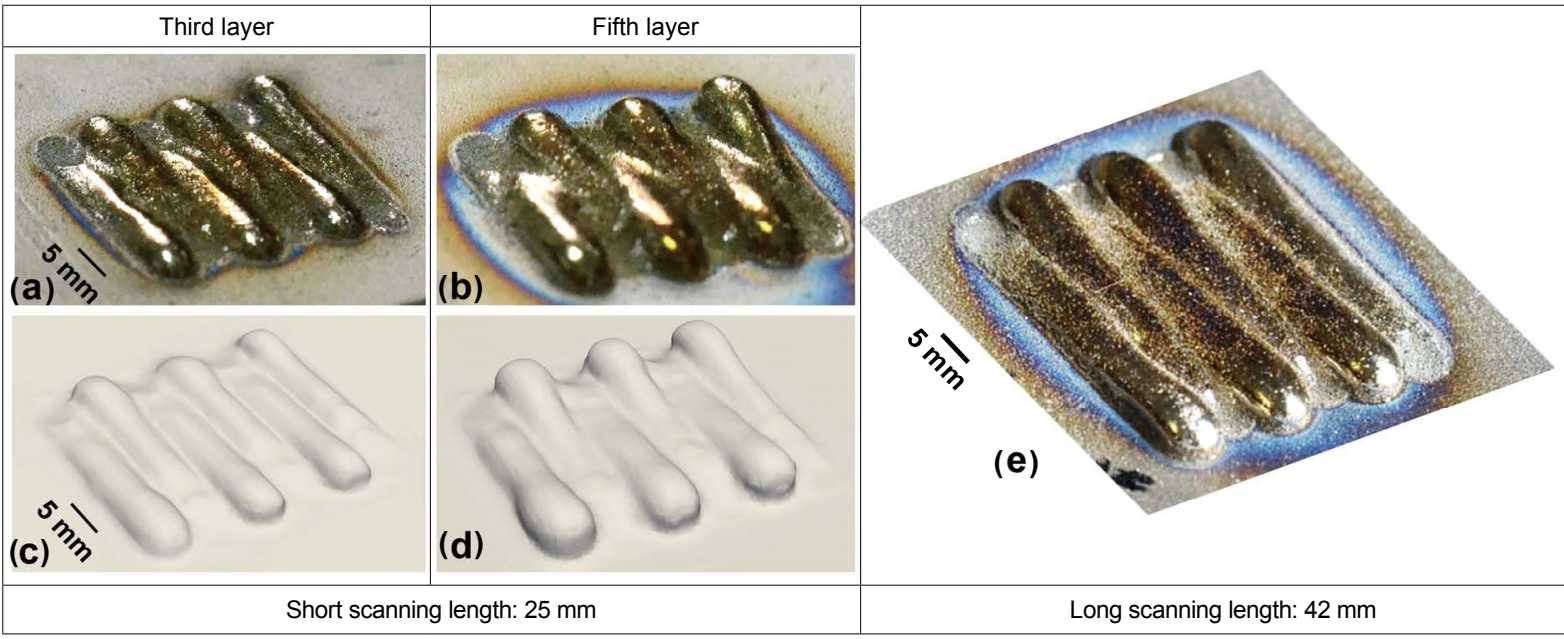

Fig. 7: Comparisons of experimental ( $a, b$ and $e)$ and computed ( $c$ and $d)$ results for five-layer and six-track laser DED of Ti-6Al-4V on top surface

(a)

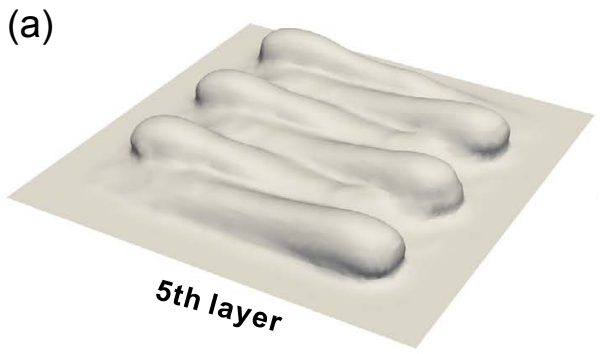

(c)

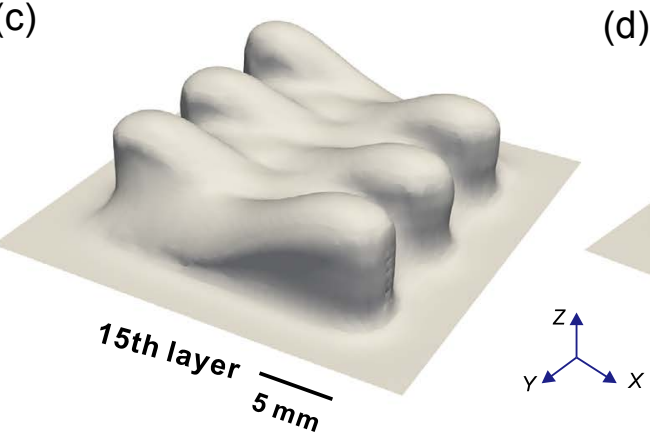

(b)

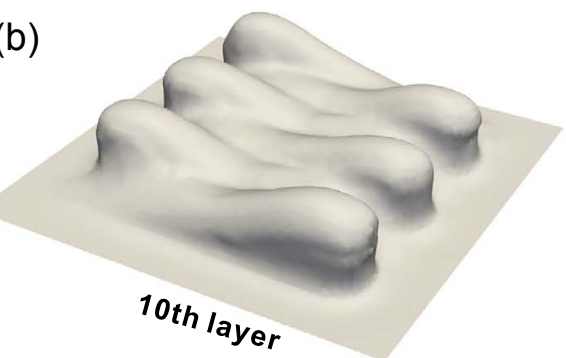

(d)

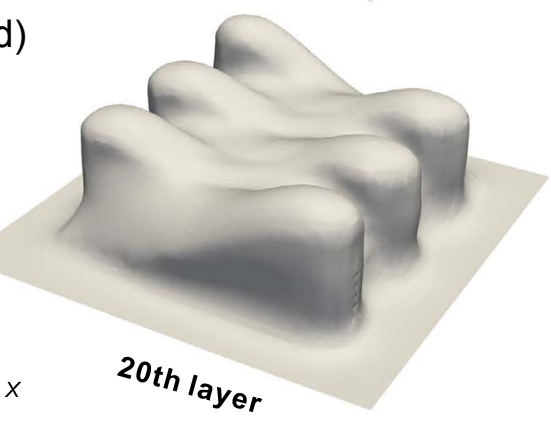

Fig. 8: Deposition profiles during multi-layer and multi-track laser DED with track length of $25 \mathrm{~mm}$

The transient deposition processes and the variations of the build profiles were systematically examined. Moreover, the printing characteristics of the builds with different scanning lengths were compared. The following conclusions can be drawn:

(1) The deposition height decreases along the scanning direction, and the height dropping rate is related to the scanning length of the tracks. The head of the track is protruding and then drops sharply along the scanning direction.

(2) The multi-layer and multi-track transient deposition result in a wavy surface profile. The degree of fluctuation is related to the scanning length. The transverse sections in the head region are symmetrically distributed. Under the influence of adjacent deposition tracks, the tracks tilt outwards and the angle increases along the scanning direction and layers.

(3) The printing process parameters should be selected appropriately for the precise shape control of small size parts.
In the case of a certain scanning length, bidirectional scanning between tracks and layers, perpendicular scanning between tracks, and periodic scanning of multiple layers can be considered for the balance of the profile variations.

\section{Acknowledgements}

This work was financially supported by the Development of a Verification Platform for Product Design, Process, and Information Exchange Standards in Additive Manufacturing (No.2019-00899-1-1), the National Natural Science Foundation of China (No. 51805267), the Natural Science Foundation of Jiangsu Province (No. BK20180483), the Fundamental Research Funds for the Central Universities (No. 30921011202), and the fund of the State Key Laboratory of Solidification Processing in NWPU (No. SKLSP201830). 


\section{References}

[1] DebRoy T, Wei H L, Zuback J S, et al. Additive manufacturing of metallic components - Process, structure and properties. Progress in Materials Science, 2018, 92: 112-224.

[2] Sames W J, List F A, Pannala S, et al. The metallurgy and processing science of metal additive manufacturing. International Materials Reviews, 2016, 61(5): 315-360.

[3] DebRoy T, Mukherjee T, Milewski J O, et al. Scientific technological and economic issues in metal printing and their solutions. Nature Materials, 2019, 18(10): 1026-1032.

[4] Knapp G L, Mukherjee T, Zuback J S, et al. Building blocks for a digital twin of additive manufacturing. Acta Materialia, 2017, 135 390-399.

[5] Wei H L, Mukherjee T, Zhang W, et al. Mechanistic models for additive manufacturing of metallic components. Progress in Materials Science, 2021, 116: 100703.

[6] Liu S, Shin Y C. Additive manufacturing of Ti6Al4V alloy: A review. Materials \& Design, 2019, 164: 107552.

[7] Gao W, Zhao S, Wang Y, et al. Numerical simulation of thermal field and Fe-based coating doped Ti. International Journal of Heat and Mass Transfer, 2016, 92: 83-90.

[8] Gan Z, Yu G, He X, et al. Numerical simulation of thermal behavior and multicomponent mass transfer in direct laser deposition of Co-base alloy on steel. International Journal of Heat and Mass Transfer, 2017, 104: 28-38.

[9] Gan Z, Yu G, He X, et al. Surface-active element transport and its effect on liquid metal flow in laser-assisted additive manufacturing. International Communications in Heat and Mass Transfer, 2017, 86: 206-214.

[10] Shin Y C, Bailey N, Katinas C, et al. Predictive modeling capabilities from incident powder and laser to mechanical properties for laser directed energy deposition. Computational Mechanics, 2018, 61(5): 617-636.

[11] Geng R W, Du J, Wei Z Y, et al. Simulation of microstructure evolution in fused-coating additive manufacturing based on phase field approach. China Foundry, 2017, 14(5): 346-352.

[12] Wolff S J, Gan Z, Lin S, et al. Experimentally validated predictions of thermal history and microhardness in laserdeposited Inconel 718 on carbon steel. Additive Manufacturing, 2019, 27: 540-551.

[13] Michopoulos J G, lliopoulos A P, Steuben J C, et al. On the multiphysics modeling challenges for metal additive manufacturing processes. Additive Manufacturing, 2018, 22: 784-799.
[14] Bai X, Colegrove P, Ding J, et al. Numerical analysis of heat transfer and fluid flow in multilayer deposition of PAW-based wire and arc additive manufacturing. International Journal of Heat and Mass Transfer, 2018, 124: 504-516.

[15] Huang Y, Khamesee M B, Toyserkani E. A new physics-based model for laser directed energy deposition (powder-fed additive manufacturing): From single-track to multi-track and multi-layer. Optics \& Laser Technology, 2019, 109: 584-599.

[16] Spranger F, Graf B, Schuch M, et al. Build-up strategies for additive manufacturing of three dimensional Ti-6Al-4Vparts produced by laser metal deposition. Journal of Laser Applications, 2018, 30(2): 1938-1387.

[17] Hu Z, Qin X, Shao T, et al. Understanding and overcoming of abnormity at start and end of the weld bead in additive manufacturing with GMAW. The International Journal of Advanced Manufacturing Technology, 2017, 95(5-8): 2357-2368.

[18] Wei H L, Liu F Q, Wei L, et al. Multiscale and multiphysics explorations of the transient deposition processes and additive characteristics during laser 3D printing. Journal of Materials Science \& Technology, 2021, 77: 196-208.

[19] Wei H L, Liu F Q, Liao W H, et al. Prediction of spatiotempora variations of deposit profiles and inter-track voids during laser directed energy deposition. Additive Manufacturing, 2020, 34 101219.

[20] Liu F Q, Wei L, Shi S Q, et al. On the varieties of build features during multi-layer laser directed energy deposition. Additive Manufacturing, 2020, 36: 101491.

[21] Shrestha S, Chou K. A study of transient and steady-state regions from single-track deposition in laser powder bed fusion. Journal of Manufacturing Processes, 2021, 61: 226-235.

[22] Wei H L, Cao Y, Liao W H, et al. Mechanisms on inter-track void formation and phase transformation during laser powder bed fusion of Ti-6Al-4V. Additive Manufacturing, 2020, 34: 101221.

[23] Fan W, Tan H, Lin X, et al. Microstructure formation of Ti-6Al-4 V in synchronous induction assisted laser deposition. Materials Design, 2018, 160: 1096-1105.

[24] Wei H L, Pal S, Manvatkar V, et al. Asymmetry in steel welds with dissimilar amounts of sulfur. Scripta Materialia, 2015, 108: 88-91.

[25] Aucott L, Dong $\mathrm{H}$, Mirihanage W, et al. Revealing internal flow behaviour in arc welding and additive manufacturing of metals. Nature Communications, 2018, 9(1): 5414. 